\title{
MULTIPLE VIEW GEOMETRY IN REMOTE SENSING: AN EMPIRICAL STUDY BASED ON PLÉIADES SATELLITE IMAGES
}

\author{
Roland Perko ${ }^{1}$, Mathias Schardt ${ }^{1}$, Livia Piermattei ${ }^{2}$, Stefan Auer ${ }^{3}$, and Peter M. Roth ${ }^{4}$ \\ ${ }^{1}$ JOANNEUM RESEARCH Forschungsgesellschaft mbH, DIGITAL, Austria \\ ${ }^{2}$ Technical University of Vienna, Department of Geodesy and Geoinformation, Austria \\ ${ }^{3}$ German Aerospace Center (DLR), Remote Sensing Technology Institute, Germany \\ ${ }^{4}$ Graz University of Technology, Institute of Computer Graphics and Vision, Austria
}

\begin{abstract}
In contrast to the fields of computer vision and photogrammetry, multiple view geometry has not been extensively exploited in the remote sensing domain so far. Therefore, an empirical study is conducted based on multi view Pléiades data that depicts a scene from multiple orbits and multiple incidence angles. First, an accuracy analysis of the 2D and 3D geo-location performance is elaborated showing that ground control points can be modelled with a root mean square residual error below $30 \mathrm{~cm}$ in East, North, and height. Second, digital surface models are reconstructed from all possible stereo pairs and are additionally fused in the multiple view geometry sense. It is shown that employing more data increases the accuracy of the digital surface model while reducing the amount of the nonreconstructed regions.
\end{abstract}

Index Terms - Multiple view geometry, digital surface model generation, Pléiades satellites, along and across track stereo.

\section{INTRODUCTION}

In airborne photogrammetry it is common sense to collect many images with high overlap both in along and in across track direction when a highly accurate $3 \mathrm{D}$ reconstruction is desired. Such highly redundant data aids the processing chain as inaccuracies or gross outliers resulting from one stereo pair can be corrected by other stereo data in the fusion process. In satellite remote sensing, however, data is mostly collected with lower redundancy due to acquisition costs, downlink capacity, and data size. Nowadays novel satellite sensors like the Pléiades constellation [1] are able to collect stereo and tri-stereo data in one single overflight, leading to redundant information in along track direction. Since the Pléiades sensors are also able to steer in across track direction they can collect images over the same scene on ground from different orbits yielding also across track stereo pairs. Such photogrammetry-like multi-overlap satellite images were, for instance, processed in [2,7]. Nonetheless, the presented tri-stereo across track data is unique in literature and the underlying potential is certainly worth being investigated. This work presents an empirical study with multiple view geometry Pléiades data (term taken from [4]). It discusses the methodologies that are needed to process such data and describes the pros and cons of using multi view data. An effect often discussed in literature is that a single stereo acquisition with an across track angle (oblique look angle orthogonal to the flight path) cause problems since then occlusions occur in this specific across direction. This issue is, for example, present in forestry applications [12] and in city modelling [9]. Having another stereo set from an adjacent or opposite orbit may solve this occlusion problem. The presented topic is scientifically motivated and there are also some drawbacks and constraints in such a data acquisition process. First, weather should be stable over the days of data collection (especially cloud free images are hard to acquire). Second, multiple stereo sets from multiple orbits result in higher data acquisition costs. Third, there might be acquisition conflicts, which may result in a priority tasking, again raising the data costs. The latter can, however also be the case for simple stereo pairs. On the other hand, such data sets may become cheaper over time or even freely available as known from other missions.

\section{INPUT DATA SETS}

To test our approach we used a data set consisting of three Pléiades stereo acquisitions from adjacent orbits over the region north to Ljubljana, Slovenia (cf. Figure 1), ground control points (GCPs) and independent check points (ICPs) measured in high resolution orthophotos, LiDAR reference digital surface model (DSM), and image coordinate measurements of the GCPs/ICPs. The whole conglomerate of data is the same as in [12]. The stereo sets were acquired within three days, the first and third set with the PHR-1A platform and the second with PHR-1B. The ellipsoidal terrain height of the region of interest ranges from 390 to 1950 meters, and the scene covers about $400 \mathrm{~km}^{2}$ consisting of agricultural land, managed forest, villages and the airport Brnik. Figure 2 depicts an orthophoto generated from image 
1 (cf. numbering in Figure 1 and in Table 1) overlaid with the locations of the 18 GCPs and 12 ICPs. Acquisition parameters are listed in Table 1 . LiDAR reference data was taken in 2015 with a mean density of 14 points $/ \mathrm{m}^{2}$ over a region of $345 \mathrm{~km}^{2}$. The LiDAR DSM with a ground sampling distance (GSD) of $1 \mathrm{~m}$ was derived using OPALS [11]. For any other detail on the dataset we refer to [12].

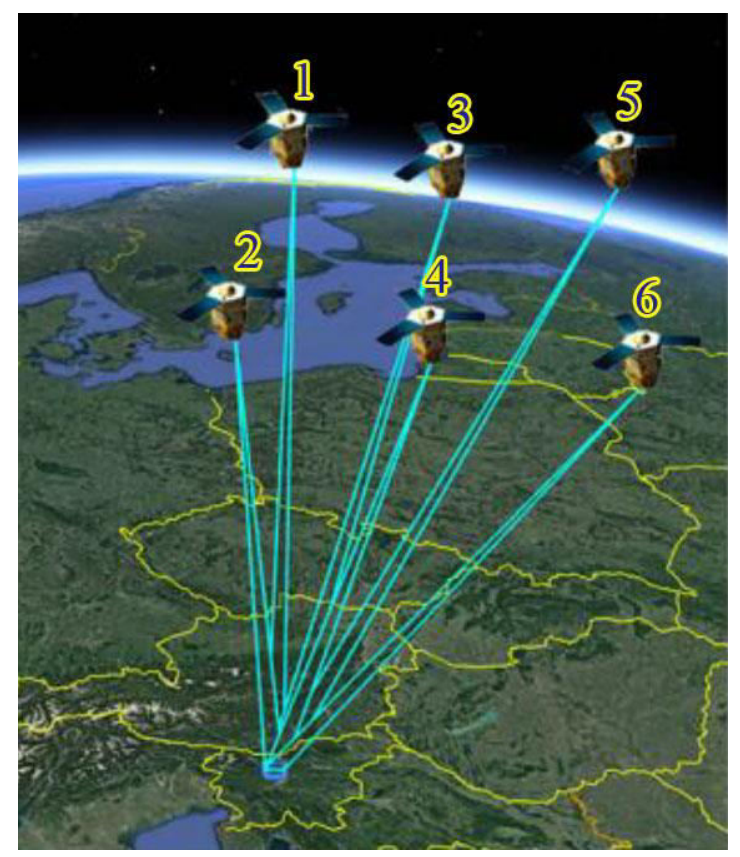

Figure 1: Study area, imaging geometries, and numbering of images of the Pléiades dataset - adapted from [12] (Google Earth preview of the footprints and the satellite's position).

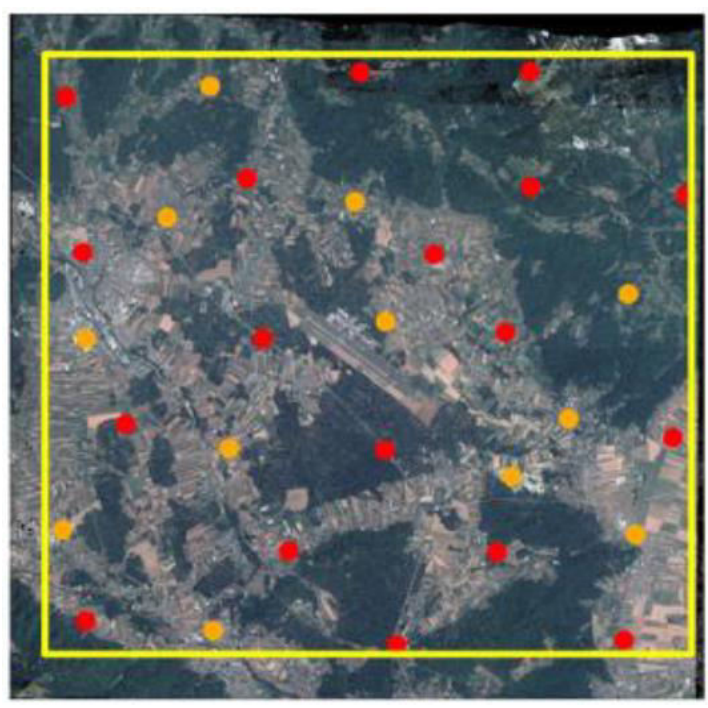

Figure 2: Orthophoto generated from the Pléiades image, visualized as true color RGB, overlaid with the GCPs (red circles) and ICPs (orange circles). The yellow rectangle represents the common regions of interest for all scene combinations - taken from [12].

\begin{tabular}{|ccccccc|}
\hline$\#$ & Date & Time & GSD $[\mathrm{m}]$ & \multicolumn{3}{c|}{ Incidence Angle $\left[{ }^{\circ}\right]$} \\
& & & along & along & across & overall \\
\hline 1 & 20130727 & $10: 10: 09$ & 0.73 & -12.12 & -1.98 & 14.9 \\
2 & 20130727 & $10: 10: 51$ & 0.74 & 12.36 & -8.59 & 12.3 \\
3 & 20130728 & $10: 03: 43$ & 0.71 & -7.39 & 9.36 & 15.3 \\
4 & 20130728 & $10: 04: 21$ & 0.74 & 14.78 & 3.95 & 11.8 \\
5 & 20130729 & $9: 55: 04$ & 0.77 & -10.20 & 22.31 & 24.1 \\
6 & 20130729 & $9: 55: 50$ & 0.78 & 16.91 & 16.90 & 23.3 \\
\hline
\end{tabular}

Table 1: Acquisition parameters of the test set.

Regarding the multiple view geometry concept our data set holds more than the three along tracks stereo pairs from descending orbit, namely 15 stereo pairs. Table 2 lists the intersection angles of all possible pairs based on the equations in [10]. Four pairs (1-3, 2-4, 3-5, 4-6) have a small intersection angle of about $12.5^{\circ}$, while all others have larger intersection angles from $23^{\circ}$ to $38^{\circ}$.

\begin{tabular}{|c|ccccc|}
\hline$\#$ & 2 & 3 & 4 & 5 & 6 \\
\hline 1 & 25.2 & 12.2 & 27.5 & 24.0 & 33.9 \\
2 & & 26.4 & 12.5 & 37.6 & 25.0 \\
3 & & & 22.7 & 13.0 & 24.7 \\
4 & & & & 30.3 & 12.6 \\
5 & & & & & 26.2 \\
\hline
\end{tabular}

Table 2: Intersection angles for all possible stereo pairs given in degrees.

\section{METHODOLOGY}

In the first step, the sensor models have to be adjusted based on the given GCPs. In the second step DSMs have to be extracted from each stereo pair and fused together. All processing is performed within the commercial software package Remote Sensing Software Graz (RSG) ${ }^{1}$, which was designed and implemented at JOANNEUM RESEARCH.

\subsection{Sensor model adjustment}

The geo-location accuracy inherent to Pléiades panchromatic imagery is reported to be $8.5 \mathrm{~m} \mathrm{CE} 90$ at nadir direction when applying the provided rational polynomial coefficient (RPC) model [1]. For validation, these RPCs are used to determine residuals, representing the initial 2D geolocation accuracy for each image. To improve the geolocation accuracy, we need to adjust the Pléiades sensor models. Therefore, we apply least squares parameter adjustment procedures based on GCPs. Only the constant and linear terms of the RPC nominators are optimized, yielding 8 parameters per image [10]. Then, the residuals are determined for the GCPs and for the ICPs that are not used in the sensor model adjustment. Next, the 3D geo-location accuracy is determined by calculating the forward point

\footnotetext{
${ }^{1}$ http://www.remotesensing.at/en/remote-sensing-software.html
} 
intersection using all sensor models and image coordinates of the GCPs / ICPs together. The 3D coordinates are then compared to the reference control points.

\subsection{DSM generation}

The employed DSM generation procedure is described in [10]. The processing chain contains more or less the standard components from photogrammetry and remote sensing: data import, epipolar rectification [3], semi-global image matching [5], forward point intersection, DSM resampling and DSM fusion [14]. A similar workflow is, for instance, also used in [12]. To get optimal results the whole process works with the original data with $0.5 \mathrm{~m} \mathrm{GSD}$. The DSM fusion is then applied with a step size of 2, thus resulting in a downsampling of factor 2 and the final DSM with $1 \mathrm{~m}$ GSD, matching to the LiDAR reference. In the DSM fusion process a $3 \times 3$ pixel kernel is used for point selection. Only if at least one third of all pixels under consideration are valid, i.e. a height value was determined in the DSM generation, also an output fused DSM value is extracted. Note, that all remaining gaps could be filled by means of interpolation within matching or fusion. However, filling is purposely omitted to be able to recognize if multi view images have an impact on the reconstruction completeness. In future, global fusion approaches that satisfy a smoothness constraint (c.f. [8, 13]) and act as outlier remover and gap filler should be applied.

\section{RESULTS}

This section first gives results of the sensor model adjustment and second on the DSM generation.

\subsection{Sensor model adjustment}

The mean values as well as the standard deviations of across and along track pixel residuals are summarized in Table 3, given for the initial and the adjusted sensor models. While most images show small initial shifts, the images 5 and 6 manifests rather large mean values, which corresponds to observations in [10]. After adjustment the standard deviations are exceptionally small, which indicates highly accurate GCPs, ICPs, and image coordinates. Overall, the residuals are larger for the ICPs that were not used in the adjustment, but still very good in comparison to previous studies [6, 10].

Table 4 shows the 3D root mean square (RMS) residuals for initial and adjusted models where all images were jointly used for point intersection. While using the initial geometries there is a systematic bias especially in North and in height, the remaining inaccuracies after adjustment are amazingly small, again due to the highly accurate reference measurements. For further processing the adjusted sensor models based on both GCPs and ICPs were used.

\begin{tabular}{|c|cccc|ccccc|}
\hline$\#$ & \multicolumn{5}{|c|}{ initial } & \multicolumn{4}{c|}{ adjusted } \\
& \multicolumn{2}{|c}{$\mu[\mathrm{pxl}]$} & \multicolumn{2}{c|}{$\sigma[\mathrm{pxl}]$} & \multicolumn{2}{c|}{$\mu[\mathrm{pxl}]$} & \multicolumn{2}{c|}{$\sigma[\mathrm{pxl}]$} \\
& across & along & across & along & across & along & across & along \\
\hline 1 & 1.68 & -0.10 & 0.68 & 0.67 & 0.05 & 0.00 & 0.42 & 0.51 \\
& 1.56 & 0.02 & 0.92 & 0.61 & -0.23 & 0.09 & 0.68 & 0.72 \\
2 & 2.85 & 2.38 & 0.85 & 0.54 & 0.08 & 0.07 & 0.40 & 0.46 \\
& 2.49 & 2.68 & 1.22 & 0.77 & -0.31 & 0.26 & 0.88 & 0.73 \\
3 & 1.54 & -0.64 & 0.69 & 0.51 & 0.04 & -0.02 & 0.45 & 0.42 \\
& 1.20 & -0.63 & 0.87 & 0.63 & -0.31 & 0.01 & 0.63 & 0.66 \\
& 1.61 & 1.60 & 0.95 & 0.82 & 0.04 & 0.04 & 0.48 & 0.67 \\
5 & 1.14 & 1.91 & 1.29 & 0.63 & -0.42 & 0.31 & 0.87 & 0.74 \\
& -2.87 & 11.72 & 0.49 & 0.42 & -0.09 & 0.32 & 0.49 & 0.37 \\
& -3.38 & 11.56 & 0.68 & 0.76 & -0.60 & 0.18 & 0.68 & 0.68 \\
& -2.82 & 12.13 & 0.47 & 0.56 & -0.09 & 0.33 & 0.47 & 0.46 \\
& -3.11 & 12.14 & 0.62 & 0.99 & -0.37 & 0.27 & 0.59 & 0.94 \\
\hline
\end{tabular}

Table 3: 2D geo-location errors w.r.t. initial and adjusted sensor models (given in pixels). For each image the first row shows the residual errors based on GCPs and the second row the residual errors based on ICPs.

\begin{tabular}{|ccccccc|}
\hline & \multicolumn{3}{c}{ initial [m] } & \multicolumn{3}{c|}{ adjusted [m] } \\
& $\mathrm{E}$ & $\mathrm{N}$ & $\mathrm{H}$ & $\mathrm{E}$ & $\mathrm{N}$ & $\mathrm{H}$ \\
\hline GCP & 0.603 & 2.577 & 3.131 & 0.208 & 0.242 & 0.244 \\
ICP & 0.618 & 2.520 & 3.350 & 0.406 & 0.395 & 0.305 \\
both & 0.609 & 2.554 & 3.220 & 0.286 & 0.301 & 0.234 \\
\hline
\end{tabular}

Table 4: 3D RMS residuals using GCPs and all images (given in meters). First, the adjustment is based on GCPs and evaluated on GCPs and ICPs. Second, adjustment is based on all control points.

\subsection{DSM generation}

For comparison, DSMs were reconstructed from all 15 possible stereo pairs. Additionally, fused DSMs based on the three along track pairs, on all pairs with intersection angles smaller than $20^{\circ}$, between $20^{\circ}$ and $30^{\circ}$, and larger than $30^{\circ}$, and on all pairs were extracted. Table 5 lists the intersection angles $(\Delta \theta)$, median (MED) and normalized median absolute deviation (NMAD) w.r.t. the LiDAR DSM and the percentages of nodata values for each model. In the comparison the whole region with a size of $345 \mathrm{~km}^{2}$ covered by LiDAR data is utilized. It can be seen that the intersection angles correlate with the amount of nodata values. Smaller angles lead to smaller occlusion areas and better matching, but also to a larger deviation from the LiDAR reference. The best accuracy (smallest NMAD) is achieved when only using pairs with large intersection angles. However, in this case only a small amount of points are reconstructed $(55.2 \%$ nodata values). Therefore, a tradeoff between accuracy (large intersection angle) and dense reconstruction (small intersection angle) is to only use stereo pairs with intersection angles in the range of $20^{\circ}$ to $30^{\circ}$ (cf. model with medium angles in Table 5). As expected the most complete reconstruction is achieved when applying all stereo pairs. It 
is also observable that larger across track angles yield to more incomplete DSMs due to occlusions.

Overall, multiple view data sets allow a better reconstruction than a single stereo pair while also increasing the completeness of the resulting DSM. To be fair, it has to be stated that the improvement of accuracy is lower than expected. Depending on the envisaged application the additional effort may be acceptable or not.

\begin{tabular}{|ccccc|}
\hline model(s) & $\begin{array}{c}\Delta \theta \\
{\left[{ }^{\circ}\right]}\end{array}$ & $\begin{array}{c}\text { MED } \\
{[\mathrm{m}]}\end{array}$ & $\begin{array}{c}\text { NMAD } \\
{[\mathrm{m}]}\end{array}$ & $\begin{array}{c}\text { nodata } \\
{[\%]}\end{array}$ \\
\hline $1-2$ & 25.2 & 0.06 & 0.97 & 14.3 \\
$1-3$ & 12.2 & -0.49 & 1.46 & 6.6 \\
$1-4$ & 27.5 & 0.07 & 0.94 & 18.8 \\
$1-5$ & 24.0 & 0.03 & 1.20 & 18.1 \\
$1-6$ & 33.9 & -0.24 & 0.86 & 27.6 \\
$2-3$ & 26.4 & 0.07 & 1.04 & 16.8 \\
$2-4$ & 12.5 & 0.08 & 1.46 & 7.8 \\
$2-5$ & 37.6 & 0.28 & 0.87 & 28.0 \\
$2-6$ & 25.0 & 0.03 & 1.14 & 22.8 \\
$3-4$ & 22.7 & 0.22 & 1.01 & 14.8 \\
$3-5$ & 13.0 & 0.43 & 1.58 & 10.7 \\
$3-6$ & 24.7 & -0.24 & 1.04 & 19.1 \\
$4-5$ & 30.3 & 0.37 & 0.93 & 25.1 \\
$4-6$ & 12.6 & -0.23 & 1.69 & 12.3 \\
5-6 & 26.2 & -0.08 & 0.92 & 21.2 \\
\hline $1-2,3-4,5-6$ & various & 0.08 & 0.84 & 12.9 \\
small angles & $<20$ & $\mathbf{- 0 . 0 1}$ & 1.14 & 11.1 \\
medium angles & $20-30$ & 0.05 & 0.93 & 7.3 \\
large angles & $>30$ & 0.16 & $\mathbf{0 . 4 1}$ & 55.2 \\
all & all & 0.05 & 1.09 & $\mathbf{1 . 1}$ \\
\hline
\end{tabular}

Table 5: Accuracy analysis of the resulting DSMs in comparison to LiDAR reference data. Best results are shown in bold face.

\section{CONCLUSIONS AND OUTLOOK}

This work deals with the aspect of multiple view geometry in remote sensing. The presented study was based on six multi view Pléiades images that depicts a scene from multiple orbits and multiple incidence angles forming along and across track stereo pairs. The analysis of the sensor models pointing precision showed very high accuracy. This could be traced back to the highly accurately measured GCPs, ICPs, and to the quality of image coordinates. Generation of DSMs was possible for all stereo pairs. It could be observed that a smaller intersection angle yields more complete reconstructions (less nodata regions), while the accuracy is lower than for pairs with larger intersection angle. Therefore, a selection of stereo pairs with medium range intersection angles represents a useful tradeoff. The fusion of all pairs resulted in the highest completeness. In future the currently employed local fusion process will be replaced by a global one.

\section{ACKNOWLEDGMENTS}

This research was partly funded by the Austrian Space Applications Programme (ASAP) through the project PleiAlps (FFG project number 859774) and the Swiss Space Office (SSO).

\section{REFERENCES}

[1] Astrium. Pleiades Technical Documents: Pleiades User Guide $\mathrm{V}$ 2.0. Technical report, https://www.intelligenceairbusds.com/en/4572-pleiades-technical-documents, 2012.

[2] G. Facciolo, C. De Franchis, and E. Meinhardt-Llopis. "Automatic 3D reconstruction from multi-date satellite images", Proc. Conf. on CVPR Workshops, pp. 57-66, 2017.

[3] KH. Gutjahr, R. Perko, H. Raggam, and M. Schardt, "The epipolarity constraint in stereo-radargrammetric DEM generation", IEEE Trans. Geosci. Remote Sens., 52, pp. 5014-5022, 2014.

[4] R. Hartley, A. Zisserman, "Multiple View Geometry in Computer Vision", Cambridge university press, 2003.

[5] H. Hirschmüller, "Stereo processing by semi-global matching and Mutual Information", IEEE Trans. on Pattern Anal. Mach. Intel., 30(2), pp. 328-341, 2008.

[6] K. Jacobsen, and H. Topan, "DEM generation with short base length Pleiades triplets", Int. Arch. Photogramm. Remote Sens. Spatial Inf. Sci., XL-3/W2, pp. 81-86, 2015.

[7] T. Krauß, P. D'Angelo, and L. Wendt. "Cross-track satellite stereo for 3D modelling of urban areas", European Journal of Remote Sensing, pp. 1-10, 2018.

[8] R. Perko and C. Zach. "Globally optimal robust DSM fusion", European Journal of Remote Sensing, 49, pp. 489-511, 2016.

[9] R. Perko, M. Hirschmugl, E. Papst, and U. Schmitt, "Mapping Singapore by Pleiades stereo data: Carbon reporting and more", in Proc. EARSeL Symposium, vol. 36, pp. 170, 2016.

[10] R. Perko, H. Raggam, M. Schardt, P.M. Roth, "Very High Resolution Mapping with the Pléiades Satellite Constellation", American Journal of Remote Sensing, vol. 6, 2, pp. 89-99, 2018.

[11] N. Pfeifer, G. Mandlburger, J. Otepka, and W. Karel, "OPALS - A framework for Airborne Laser Scanning data analysis", Comput. Environ. Urban Syst., 45, pp. 125-136, 2014.

[12] L. Piermattei, M. Marty, W. Karel, C. Ressl, M. Hollaus, C. Ginzler, and N. Pfeifer, "Impact of the Acquisition Geometry of Very High-Resolution Pléiades Imagery on the Accuracy of Canopy Height Models over Forested Alpine Regions", Remote Sensing, 10(10), 1542, 2018.

[13] T. Pock, L. Zebedin, and H. Bischof (2011) - Rainbow of computer science. Chapter "TGV-fusion", pp. 245-258, SpringerVerlag, Berlin, Heidelberg, 2011.

[14] M. Rumpler, A. Wendel, and H. Bischof, "Probabilistic range image integration for DSM and true-orthophoto generation", in Proc. Scandinavian Conf. on Image Analysis, pp. 533-544, 2013. 